\title{
Quimioterapia metronómica en pacientes con cáncer de mama metastásico
}

\section{Metronomic chemotherapy in patients with metastatic breast cancer}

\author{
Jorge Soriano-Lorenzo ${ }^{1, a}$, Jorge Soriano Garcia ${ }^{2, b}$, Mayte Lima Pérez ${ }^{2, c}$ \\ ${ }^{1}$ Facultad de Medicina "Manuel Fajardo", Universidad de Ciencias Médicas de la Habana. La Habana, Cuba. \\ ${ }^{2}$ Servicio de Oncología Clínica, Hospital Clinico Quirúrgico Hermanos Ameijeiras. La Habana, Cuba. \\ a Estudiante de medicina, ORCID: https://orcid.org/0000-0003-2211-5696 \\ ${ }^{b}$ Especialista en oncologia, doctor en ciencias médicas. ORCID: https://orcid.org/0000-0002-7713-5750 \\ ${ }^{c}$ Especialista en oncologia, ORCID: https://orcid.org/0000-0001-6607-3913
}

\section{An Fac med. 2020;81(1):80-6 / DOI: https://doi.org/10.15381/anales.v81i1.17786}

\section{Correspondencia:}

Jorge Soriano Lorenzo

sorianolorenzo@infomed.sld.cu

Recibido: 13 de febrero 2020

Aceptado: 18 de marzo 2020

Publicación en línea: 31 de marzo 2020

Conflictos de interés: Los autores declaran no tener conflictos de interés.

Fuente de financiamiento:

Autofinanciado

\section{Citar como: Soriano-Lorenzo J,}

Soriano J, Lima M. Quimioterapia metronómica en pacientes con cáncer de mama metastásico. An Fac med. 2020;81(1):80-6. DOI: https://doi. org/10.15381/anales.v81i1.17786

\section{Resumen}

La quimioterapia metronómica es un tipo de quimioterapia cuya toxicidad es mínima. Consiste en administrar agentes antineoplásicos habituales a intervalos muy próximos uno del otro y a dosis relativamente bajas durante un tiempo prolongado y sin periodos de descanso farmacológico. Esta terapia reduce la toxicidad y elimina la necesidad de tratamientos de soporte, por lo tanto, el empleo de esta modalidad de tratamiento en pacientes con enfermedad metastásica resulta de gran interés. Muchas investigaciones han evaluado la administración de medicamentos antineoplásicos en esquemas metronómicos, tanto en monoterapia como en combinación. Los fármacos mayormente estudiados son la ciclofosfamida, vinorelbina y capecitabina. Diversos estudios han mostrado la eficacia y tolerancia de esta terapia combinada con la hormonoterapia e inmunoterapia. Es necesaria mayor evidencia cientifica para definir cuestiones como: pacientes que más beneficio puedan obtener y las combinaciones terapéuticas a emplear de acuerdo al tipo de paciente.

Palabras clave: Administración Metronómica; Quimioterapia; Neoplasias de la Mama; Antineoplásicos (fuente: DeCS BIREME).

\section{Abstract}

Metronomic chemotherapy is a type of chemotherapy whose toxicity is minimal. It consists of administering usual antineoplastic agents at intervals very close to each other and at relatively low doses for a prolonged time without periods of pharmacological rest. This therapy reduce toxicity and eliminates the need for supportive treatments, therefore, the use of this treatment modality in patients with metastatic disease is of great interest. Many investigations have evaluated the administration of antineoplastic drugs in metronomic schemes, both in monotherapy and in combination. The most studied drugs are cyclophosphamide, vinorelbine and capecitabine. Several studies have shown the efficacy and tolerance of this therapy combined with hormone therapy and immunotherapy. More scientific evidence is needed to define issues such as: patients who can obtain more benefit and the therapeutic combinations to be used according to the type of patients.

Keywords: Administration, Metronomic; Drug Therapy; Breast Neoplasms; Antineoplastic Agents (source: MeSH NLM). 


\section{INTRODUCCIÓN}

En el desarrollo de los agentes antineoplásicos el efecto citotóxico sobre las células tumorales se encuentra relacionado con la dosis de estos agentes y constituye una de las principales causas de su eficacia. De hecho, el principio de la intensidad de la dosis está respaldado por varios modelos experimentales donde un mínimo incremento en las dosis de los fármacos eleva la muerte de células tumorales ${ }^{(1)}$

En las pacientes con cáncer de mama metastásico (CMM) la administración de quimioterapia citotóxica a dosis máximas tolerables (DMT) tiene un beneficio modesto en prolongación de supervivencia y trae consigo un aumento significativo de las toxicidades, lo cual deteriora la calidad de vida de las pacientes.

Se ha demostrado que la administración frecuente de dosis bajas de agentes citotóxicos, sin pausas extendidas, puede no solo ser tan eficaz como la administración de quimioterapia a DMT, sino que además resulta menos tóxica ${ }^{(2,3)}$.

La quimioterapia metronómica (QTM) es un tipo de quimioterapia cuya toxicidad es mínima. Consiste en la administración de agentes antineoplásicos habituales a intervalos muy próximos uno del otro y a dosis relativamente bajas durante un tiempo prolongado y sin periodos de descanso farmacológico ${ }^{(4)}$. Los modelos experimentales preclínicos han demostrado que su eficacia antineoplásica se basa en sus efectos antiangiogénicos tanto a nivel local como sistémico. También se ha sugerido la elevación de los niveles de trombospondina 1 un potente inhibidor específico endotelial de la angiogénesis como mecanismo responsable de estos efectos antiangiogénicos $(2,3)$. Una de las principales ventajas de la QTM es la de retardar el comienzo de mecanismos de resistencia adquirida a fármacos dependientes de mutaciones, ya que en este caso el blanco terapéutico son las células endoteliales, que poseen una gran estabilidad genética ${ }^{(5,6)}$.

El objetivo del presente artículo es realizar una revisión sobre los conocimientos y resultados disponibles hasta la actualidad del uso de la quimioterapia metronómica en pacientes con CMM.

\section{METODOLOGÍA DE BÚSQUEDA Y SELECCIÓN DE RESULTADOS}

Se muestra una revisión de los resultados disponibles hasta la actualidad sobre el empleo de la quimioterapia metronómica en pacientes con cáncer de mama. Para ello se realizó una búsqueda en la base de datos bibliográficos de PubMed y Scielo. Los términos de búsqueda fueron "cáncer de mama metastásico y quimioterapia metronómica", "quimioterapia metronómica e inmunoterapia" "quimioterapia metronómica y terapia hormonal". En la búsqueda se obtuvo un total de 920 artículos. Se incluyeron estudios originales, artículos de revisión y revisiones sistemáticas. Los resultados fueron obtenidos en idioma inglés y español. Los artículos se evaluaron por su título y resumen. Los artículos de interés fueron revisados a texto completo ${ }^{(74)}$. Se encontraron artículos que no fueron evaluados por no estar disponibles a texto completo. Se excluyeron reportes de casos, artículos no disponibles a texto completo y artículos cuyo título y resumen no respondían a los objetivos de la presente revisión.

\section{DESARROLLO DEL TEMA}

Quimioterapia metronómica con esquemas de monoterapia o combinados

El primer estudio sobre QTM en pacientes con CMM fue publicado en el año $2002{ }^{(7)}$. Los primeros estudios incluían pacientes con estado funcional muy deteriorado y/o fuertemente pre-tratados, mientras que los más recientes emplean esta modalidad como primera o segunda línea terapéutica.

Dentro de los fármacos más estudiados en este tipo de quimioterapia se encuentra la ciclofosfamida. La administración de ciclofosfamida en regímenes metronómicos no solo ha mostrado actividad antiangiogénica, sino que también ha mostrado inducir reducción de las células $T$ regulatorias circulantes, asociado a una supresión de las funciones inhibitorias en las células T y NK, lo cual permite restaurar la proliferación de las células $T$ periféricas y reduce la tolerancia inmune inducida por el tumor ${ }^{(8)}$.
Colleoni et al (7), en un estudio con 63 pacientes diagnosticadas de CMM las cuales habían recibido tratamiento previo para su enfermedad, estudió la combinación de ciclofosfamida y metotrexate, y su relación con los niveles de factor de crecimiento del endotelio vascular (VEGF). Con el empleo de este régimen se logró una respuesta objetiva (RO) en el 19\% (95\% Cl 10,2\%-30,9\%) de las pacientes y un beneficio clínico (BC) de 31,7\% (95\% Cl 20,6\% - 44,7\%). Los anteriores resultados se obtuvieron en ausencia de toxicidades significativas. También se observaron reducciones en los niveles de VEGF, los cuales fueron significativos en los pacientes que seguían en tratamiento luego de 6 meses $(p<0,001)$. Gebbia et al ${ }^{(9)}$, en un estudio retrospectivo incluyó un total de 61 pacientes con enfermedad avanzada, las cuales mostraron resistencia a tratamiento hormonal y enfermedad progresiva luego de empleo de quimioterapia; estos pacientes fueron distribuidos en 2 grupos, en el grupo 1, se administró ciclofosfamida y en el grupo 2 , se empleó la terapia combinada con metotrexate. Este estudio no mostró diferencia significativa de RO (Grupo 1: 14\% vs Grupo 2: $20 \% ; p=0,45)$ entre ambos grupos. La media de duración de la respuesta fue discretamente mayor en los pacientes tratados con terapia combinada (Grupo 1: 5,2 meses vs Grupo 2: 6,2 meses), en términos de supervivencia global (SG) no se mostró tampoco diferencias significativas (Grupo 1: 12,8 meses vs Grupo 2: 14 meses). En este estudio se observó que la presencia de metástasis hepática estaba asociada a peores resultados en cuanto a RO y supervivencia. Estos datos sugieren que la prescripción de la QTM puede ser más apropiada para pacientes con enfermedad metastásica sin predominio visceral. Similar a estos resultados, Soriano et al ${ }^{(10)}$, en un estudio prospectivo con 28 pacientes con enfermedad en progresión en las que se empleó la combinación de ciclofosfamida y metotrexate, reportó que aquellas pacientes con mayor número de sitios metastásicos (>2) no obtenían una respuesta terapéutica a este tipo de quimioterapia, lo cual pudiera relacionarse con factores como: resistencia adquirida por mecanismo de remodelación vascular, crecimiento tumoral independientemente de mecanismos angiogéni- 
cos e incremento de tolerancia tumoral a la hipoxia. Por otra parte, el mayor número de pacientes que respondían a la terapia correspondían a pacientes con receptores hormonales $(\mathrm{RH})$ positivos, similar a lo obtenido en otros estudios que asocian esta condición a una mayor estabilización de la enfermedad, menor proliferación, índice replicativo y grado tumoral ${ }^{(11,12)}$

La administración de vinorelbina en regímenes metronómicos ha sido ampliamente estudiada, el índice de RO a este régimen es de alrededor de un $38 \%$ y su índice de BC varía desde un $42 \%$ hasta $62 \%{ }^{(13)}$. En un estudio fase II $^{(14)}$ con 34 pacientes ancianas diagnosticadas de CMM en los cuales se empleó vinorelbina como primera línea de tratamiento, se obtuvo una RO en un 38\% (IC 95\%: 28-48\%), en un $29 \%$ se logró una estabilización de la enfermedad, lográndose un BC de al menos 12 semanas en el 68\% (IC 95\%: 60,7$81,9 \%)$. La mediana de supervivencia libre de progresión (SLP) y supervivencia global (SG) fue de 7,7 meses (IC 95\%: 6,9- 9,05 meses) y 15,9 meses (IC 95\%: 13,1-15,91 meses) respectivamente.

La capecitabina es otro fármaco estudiado en esquemas metronómicos para el CMM. En un estudio fase II, Fedele at al (15), incluyó 60 pacientes las cuales habían recibido previamente al menos un régimen de tratamiento convencional para su enfermedad metastásica. En este grupo de pacientes se logró una RO de $24 \%$ (IC 95\%: $13 \%$ - 35\%), se obtuvo además un BC de más de 24 semanas en el 62\% (IC 95\%: $50 \%-74 \%$ ), en términos de supervivencia se logró una mediana de SLP y SG de 7 y 17 meses, respectivamente. Con respecto a los eventos adversos desarrollados, se observó una buena tolerancia al tratamiento, siendo la diarrea y el síndrome manos-pies los más frecuentes. Ningún caso requirió reducción de la dosis o interrupción del tratamiento. Otro estudio llevado a cabo en 323 pacientes por Stockler et al ${ }^{(16)}$, comparó tres regímenes diferentes: capecitabina administrada de forma intermitente a $1000 \mathrm{mg} / \mathrm{m}^{2} / 2$ veces al día por 14 días cada 21 días, capecitabina administrada de forma continuada a $650 \mathrm{mg} /$ $\mathrm{m}^{2}$ (días $1-14$ ) y ciclofosfamida $100 \mathrm{mg} / \mathrm{m}^{2}$ junto metotrexate $40 \mathrm{mg} / \mathrm{m}^{2}$ y 5 -fluouracilo $600 \mathrm{mg} / \mathrm{m}^{2}$ (días 1 y 8 cada 28 días). Este estudio demostró que ambos esquemas de capecitabina eran tan eficaces como la combinación de ciclofosfamida junto a metotrexate y 5-fluouracilo (CMF). Taguchi et al ${ }^{(17)}$, en un estudio fase II llevado a cabo en 33 pacientes las cuales fueron tratadas con capecitabina, obtuvo una mediana de SLP y SG de 6,9 y 24,8 meses respectivamente, manteniendo una adecuada tolerancia al tratamiento.

Debido a la gran eficacia terapéutica en esquemas metronómicos de estos fármacos en el contexto de la enfermedad metastásica, varios estudios han investigado la eficacia y tolerancia de la combinación entre ambos. En el año 2012, Saridakis et al ${ }^{(18)}$, en un estudio fase I desarrollado con el objetivo de determinar la dosis limitante de vinorelbina en combinación con capecitabina en esquema metronómicos, incluyó un total de 36 pacientes a los cuales se les administró este tratamiento. La dosis inicial de vinorelbina fue de $30 \mathrm{mg}$ cada 2 días con incrementos de $10 \mathrm{mg}$ en cohortes de pacientes, la capecitabina fue administrada con una dosis inicial de $800 \mathrm{mg} / \mathrm{m}^{2}$ dos veces al día durante 14 días en ciclos de 21 días. Luego de 8 incrementos de la dosis, el límite de la combinación fue de $70 \mathrm{mg}$ para la vinorelbina y $1250 \mathrm{mg} / \mathrm{m}^{2}$ para la capecitabina, las toxicidades que limitaron la dosis fue la neutropenia febril grado 3-4 y la diarrea grado 4. En este estudio se recomendaba que las dosis máximas tolerables de la combinación eran de 60mg para la vinorelbina y $1250 \mathrm{mg} / \mathrm{m}^{2}$ para la capecitabina. El estudio VICTOR-1 fase I/ Il llevado a cabo por Cazzaniga et al (19), estableció la dosis máxima tolerable de vinorelbina para combinar con capecitabina en 40 mg administrados los días 1 , 3 y 5 de cada semana. En este estudio se trataron 34 pacientes con la combinación de vinorelbina $40 \mathrm{mg}$ y capecitabina 500 mg tres veces al día. Con estas dosis la mayoría de los eventos adversos fueron grado 1. En estos pacientes se obtuvo una RO de $45,1 \%$ y un BC de 24 semanas y más en el $58,1 \%$. Posteriormente en el estudio multicéntrico VICTOR-2 fase II (20) desarrollado por el mismo grupo con el objetivo de confirmar los resultados obtenidos en el estudio anterior, se incluyeron 80 pacientes en los cuales se administró la misma terapia. Esta combinación se administró como primera línea de tratamiento a 35 pacientes $(43,8 \%)$. Se obtuvo un BC de al menos 24 semanas en el $48,8 \%$, este BC fue mayor en pacientes en los que se empleó este tratamiento como segunda línea de tratamiento (51,1\%; IC 95\%: 35,8\%-66,3\%) en comparación con los que se empleó como primera línea (45,7\%; IC 95\%: 28,8\%-63,4\%). Con respecto a la expresion de $\mathrm{RH}$ se obtuvo un mayor $\mathrm{BC}$ en aquellos pacientes con $\mathrm{RH}$ positivos (55,8\%; IC 95\%: 41,3\%- 69,5\%) que en las pacientes con receptores triple negativo (35,7\%; IC 95\%: 18,6\%- 55,9\%), con respecto al sitio metastásico el $\mathrm{BC}$ fue mayor en las pacientes sin metástasis viscerales (59,1\%; IC 95\%: 36,4\%- 79,3\%) que en aquellas con enfermedad metastásica con compromiso visceral (44,8\%; IC 95\%: 31,7\%-58,5\%). Globalmente se obtuvo una RO de 29,7\% (IC 95\%: 19,7\% $-41,5 \%)$; en el grupo de pacientes en los que se empleó este tratamiento como primera línea se obtuvieron mayores porcentajes de RO (35,5\%; IC 95\%: 19,2\% - 54,6\%) y de ICE (74,2\%; IC 95\%: 55,4\% - $88,1 \%)$ que en aquellos que se empleó como segunda línea de tratamiento ( $\mathrm{RO}=$ 25,6\%; IC 95\%: $13,5 \%$ - 41,2\%) (ICE= 67,4\%; IC 95\%: 51,5\%-80,9\%). La mediana de duración de la RO fue de 8,2 meses (IC 95\%: 5,2-12,8), la cual fue mayor en los pacientes que recibieron esta modalidad como primera línea de tratamiento (11,3 meses vs. 6,4 meses). Respecto a la SLP, se observó una mediana de 7,5 meses (IC 95\%: 3,7- 11,5), la cual fue discretamente mayor en los tratados como primera línea (7,9 meses vs. 7,2 meses). De acuerdo con la expresion de $\mathrm{RH}$, aquellas pacientes con $\mathrm{RH}$ positivos mostraron una SLP mayor que aquellas con receptores triple negativos (8,3 meses vs 6,5 meses). En este estudio se mostró una adecuada tolerancia por las pacientes.

La eficacia terapéutica y seguridad de la combinación de vinorelbina junto a ciclofosfamida y capecitabina fue evaluada por Montagna et al ${ }^{(21)}$, en un ensayo fase II con 108 pacientes diagnosticadas de CMM y RH positivo, los cuales fueron divididos en 2 grupos (no pretratados: 43 pacientes y grupo de pretratados: 65 pacientes). Se obtuvo un BC mayor en los pacientes que no habían recibido previamente tratamiento que en aque- 
llos que habían sido pretratados (81\% vs $74 \%$ ). Con respecto a la SLP, hubo una diferencia significativa $(p=0,004)$ entre el grupo de los pacientes que previamente no habían sido tratados y el grupo previamente tratado $(25,1$ meses vs 11,2 meses). La SG a 2 años, aunque fue mayor en el grupo de los pacientes que no habían sido previamente tratados $(91 \%$ vs $83 \%)$ no mostró diferencias significativas $(p=0,887)$. Con respecto a los eventos adversos, los pacientes presentaron una buena tolerancia, solamente se reportaron 2 casos en los cuales hubo que detener el tratamiento. En un estudio derivado de este ensayo, Montagna et al ${ }^{(22)}$, evaluó la asociación de la infiltración linfocítica y el pronóstico de las pacientes con CMM con $\mathrm{RH}$ positivos tratadas con QTM. De los 108 pacientes que fueron incluidos inicialmente en el estudio, solamente 92 presentaban suficiente tejido tumoral para la evaluación de la infiltración linfocitaria. Se encontró una correlación significativa entre el nivel de infiltración linfocitaria y los altos niveles de Ki-67. Mediante un análisis multivariado, el incremento de la infiltración linfocitaria se asociaba a peores resultados de SLP (HR: 1,27; $p=0,008$ ). Al dividir a los pacientes en tres categorías de acuerdo al nivel de infiltración linfocitaria (0-4\%; $5-9 \%$; $>10 \%)$ se observaron diferencias significativas de SLP. Este estudio abre una posible puerta para el uso de los niveles de infiltración linfocitaria como biomarcador en las pacientes con CMM luminal que serán tratadas con QTM.

Otros estudios (Tabla 1) han evaluado la eficacia de otras combinaciones como la ciclofosfamida junto a la capecitabina ${ }^{(23)}$ y la vinorelbina junto a temozolamida (24).

Quimioterapia metronómica en combinación con terapias endocrinas

La baja toxicidad de la QTM ha despertado un gran interés por la combinación de esta con otras modalidades terapéuticas como la hormonoterapia (Tabla 2). Así, en un estudio aleatorizado fase II (25), se incluyó un grupo de 114 mujeres de edad avanzada, las cuales fueron distribuidas aleatoriamente a recibir tratamiento primario con letrozol o en combinación con ciclofosfamida. La RO fue de $71,9 \%$ (IC 95\%: 60\%- 83,3\%) en las pa-
Tabla 1. Estudios que han empleado quimioterapia metronómica con fármacos antineoplásicos en monoterapia o combinados.

\begin{tabular}{lcccccc} 
Autor (año) & $\mathbf{n}$ & $\begin{array}{c}\text { Esquema } \\
\text { empleado }\end{array}$ & RO & BC & $\begin{array}{c}\text { SLP } \\
\text { (meses) }\end{array}$ & $\begin{array}{c}\text { SG } \\
\text { (meses) }\end{array}$ \\
\hline Colleoni, et al ${ }^{(7)}(2002)$ & 63 & CTX + MTX & $19 \%$ & $31,7 \%$ & - & - \\
\hline Gebbia et al ${ }^{(9)}(2012)$ & 61 & CTX & $14 \%$ & - & 3,8 & 12,8 \\
\cline { 2 - 7 } & & CTX + MTX & $20 \%$ & - & 4,2 & 13,8 \\
\hline Soriano et al $^{(10)}(2009)$ & 28 & CTX + MTX & $49,9 \%$ & $61,1 \%$ & - & - \\
\hline Mahmound $^{(11)}(2017)$ & 48 & CTX + MTX & $25 \%$ & - & 5 & 7 \\
\hline Krajnak $^{(12)}(2018)$ & 35 & CTX + MTX & $28,5 \%$ & - & 3 & - \\
\hline Addeo $^{(14)}(2010)$ & 34 & VNR & $38 \%$ & - & 7,7 & 15,9 \\
\hline Fedele $^{(15)}(2012)$ & 60 & CAPE & $24 \%$ & $62 \%$ & 7 & 17 \\
\hline Taguchi $^{(17)}(2010)$ & 33 & CAPE & $18 \%$ & $42 \%$ & 6,9 & 24,8 \\
\hline Cazzaniga $^{(19)}(2014)$ & 34 & VNR+CAPE & $45,1 \%$ & $58,1 \%$ & 18,5 & - \\
\hline Cazzaniga $^{(20)}(2016)$ & 80 & VNR+CAPE & $29,7 \%$ & $48,8 \%$ & 7,5 & - \\
\hline Yoshimoto $^{(23)}(2012)$ & 45 & CTX+CAPE & $44 \%$ & $57,8 \%$ & 12,3 & - \\
\hline Addeo $^{(24)}(2012)$ & 32 & VNR+TMZ+RT & $52 \%$ & $77 \%$ & 8 & 11 \\
\hline
\end{tabular}

BC: beneficio clínico; CAPE: capecitabina; CTX: ciclofosfamida; MTX: metotrexate; RO: respuesta objetiva; RT: radioterapia, SG: supervivencia global; SLP: supervivencia libre de progresión; VNR: vinorelbina cientes tratadas con letrozol y de $87,7 \%$ (IC 95\%: 78,6\%- 96,2\%) en las que se empleó la terapia combinada. En el grupo de las pacientes tratadas con la combinación de hormonoterapia más QTM se observó disminución en la expresion de Ki-67 y del factor de crecimiento del endotelio vascular-A (VEGF-A).

Otro estudio fase II ${ }^{(26)}$ evaluó la seguridad y eficacia terapéutica de la combinación del megestrol y ciclofosfamida. Este estudio incluyó a 29 pacientes postmenopáusicas las cuales habían recibido otras terapias con anterioridad. La RO obtenida fue de $31 \%$ y la media de SG fue de 13,4 meses.

La combinación de fulvestrant junto con capecitabina fue evaluada por Schwartzberg et al ${ }^{(27)}$, en un estudio que incluyó 41 pacientes con CMM, RH positivos y HER2 negativo. En este estudio la dosis de capecitabina fue administrada según el peso de las pacientes, para aquellas pacientes con un peso inferior a los $80 \mathrm{Kg}$ se administró una dosis de 1500 $\mathrm{mg} /$ día, y en las que superaban este peso $2000 \mathrm{mg} /$ día. El fulvestrant fue administrado a una dosis de 500 mg el primer día, seguido de una dosis de $250 \mathrm{mg}$ el día 15 y 29, para luego continuar con una dosis de 250 mg cada 28 días. Este estudio registró una mediana de SLP y SG de 14,9 y 28,6 meses respectivamente, con una adecuada tolerancia. En un estudio retrospectivo desarrollado por Aurilio et al ${ }^{(28)}$, se mostró una prolongación del BC en las pacientes que recibieron terapia combinada de fulvestrant junto a ciclofosfamida y metotrexate.

La combinación de la terapia endocrina y la quimioterapia metronómica en la práctica clinica diaria debe ser bien indicada y no se recomienda en todos los casos, ya que no existe evidencia de que la adición de quimioterapia a la terapia endocrina produzca beneficios clínicos cuando la paciente presenta todavía una enfermedad endocrino-sensible ${ }^{(29)}$.
Tabla 2. Estudios que han empleado combinación de quimioterapia metronómica con terapia endocrina.

\begin{tabular}{lcccc}
\hline Autor (año) & $\mathbf{n}$ & Esquema empleado & RO & BC \\
Bottini $^{(25)}(2006)$ & 114 & CTX + LET & $71,9 \%$ & - \\
\hline Lichetta $^{(26)}(2010)$ & 22 & CTX + MGA & $31 \%$ & $41,3 \%$ \\
\hline Schwartzberg $^{(27)}(2014)$ & 41 & CAPE + FLV & $24,4 \%$ & $58,5 \%$ \\
\hline
\end{tabular}

BC: Beneficio clínico; RO: Respuesta Objetiva. CAPE: capecitabina; CTX: ciclofosfamida; FLV: fulvestrant; MGA: megestrol 
Quimioterapia metronómica en combinación con terapias biológicas

En diversos estudios se ha demostrado la acción de la terapia metronómica como potenciador de la respuesta inmune antitumoral ${ }^{(30,31)}$. Se ha propuesto que la QTM puede disminuir el estatus inmunosupresor del huésped a través de al menos tres mecanismos: 1) aumentando la función de las células dendríticas en la presentación de los antígenos tumorales, 2) aumentando la producción de células $T$ reactivas y 3) disminuyendo la inmunosupresión desarrollada por el tumor. Al realizar una combinación de esta con la inmunoterapia, es de esperar un aumento de los efectos anti-tumorales de la inmunoterapia ${ }^{(31)}$. Por tanto, la combinación de estas terapias es una estrategia atractiva que puede aumentar la efectividad terapéutica.

García-Sáenz et al ${ }^{(32)}$, evaluó la combinación de ciclofosfamida y metotrexate junto con el bevacizumab en pacientes refractarias a taxanos y antraciclinas. Este estudio incluyó 22 pacientes y reportó un BC en el $63 \%$ de las pacientes. La mediana de SLP y SG fue de 7,5 y 13,6 meses, respectivamente. Los anteriores resultados se obtuvieron con leves índices de toxicidad. Por otra parte, Dellapasqua et al ${ }^{(33)}$, en un estudio fase II con 46 pacientes evaluó la eficacia de la combinación de capecitabina y ciclofosfamida junto a bevacizumab. La RO se logró en un $48 \%$ de los pacientes y el BC fue de $69 \%$.

Otros fármacos como los inhibidores del receptor de crecimiento epidérmico (EGFR) han sido empleados en combinación con esquemas metronómicos. Así, Montagna et al ${ }^{(34)}$, en un estudio fase II evaluó la efectividad y seguridad de la combinación de capecitabina y ciclofosfamida junto a bevacizumab y erlotinib en 24 pacientes que no habían recibido previamente tratamientos para su enfermedad metastásica. Con el empleo de esta combinación obtuvo una RO en el 62\% (IC 95\%: 41\% - 48\%) y un BC de más de 24 semanas en el 75\% (IC 95\%: 53\%90\%). La mediana de SLP y SG fue de 43 (IC 95\%: 21- 69) y 108 meses (IC 95\%: 70 - 110) respectivamente. La tolerancia fue adecuada, la mayoría de los eventos adversos fueron grados 1-2. En este estudio se observó una diferencia significativa en cuanto a SLP entre los pacientes que presentaron niveles de células progenitoras endoteliales circulantes (CEP's) $(p=0,044)$ $y$ de VEGFR $(p=0,027)$ más bajos al inicio del tratamiento con respecto a aquellos que presentaron niveles elevados. Estos resultaron contrastaban con los obtenidos por Dellapasqua et al ${ }^{(33)}$ y Calleri et al (35), donde los valores más bajos de CEP's y VEGFR al inicio de la terapia se relacionaba con mejores resultados terapéuticos, por lo tanto, podrían ser empleados como método de predicción.

La combinación de QTM junto con trastuzumab ha sido estudiada en pacientes con sobreexpresión de HER2 ${ }^{(29)}$. Orlando et al (36), evaluó la administración de la combinación de ciclofosfamida y metotrexate junto con trastuzumab en pacientes con CMM y sobreexpresión de HER2. De las 22 pacientes incluidas en el estudio se obtuvo una RO en un $18 \%$, el BC luego de 24 semanas fue $46 \%$. Esta terapia mostró buena tolerancia, las toxicidades más registradas fueron la leucopenia y la elevación de los niveles de las transaminasas. Recientemente se publicaron los resultados del ensayo clínico multicéntrico aleatorizado fase II EORTC 75111-10114 ${ }^{(37)}$, en este estudio incluyó 80 pacientes con CMM, sobreexpresión de receptor HER2, edad>70 años y que no hubieran recibido tratamiento previo para su enfermedad metastásica. Las pacientes fueron distribuidas al azar a recibir doble bloqueo con trastuzumab y pertuzumab (39 pacientes) o trastuzumab y pertuzumab en combinación con ciclofosfamida a dosis metronómicas (41 pacientes). Luego de una mediana de seguimiento de 20,7 meses, se observó una SLP de 5,6 meses (IC 95\%: 3,6-16,8) en las pacientes tratadas con trastuzumab y pertuzumab y de 12,7 (IC 95\%: 6,7-24,8) meses, en las que se empleó la combinación de trastuzumab y pertuzumab junto con ciclofosfamida. En cuanto a las toxicidades presentadas, los eventos grado 3-4 más frecuentemente registrados fueron la hipertensión, diarrea, fatiga, dolor y eventos tromboembólicos; con excepción de este último en todos los demás se observaron aproximadamente en la misma cantidad de pacientes en ambos grupos, los eventos tromboembólicos fue- ron más frecuentes en el grupo tratado con la combinación de trastuzumab, pertuzumab y ciclofosfamida (0 vs 4). Este estudio propone que la combinación de la QTM con ciclofosfamida junto a trastuzumab y pertuzumab, puede ser una opción para pacientes mayores de 70 años, y que el trastuzumab-emtansine puede emplearse luego de objetivar progresión con este esquema y de esta forma sustituir la necesidad de la quimioterapia con taxanos en esta población.

La combinación de la QTM junto a vacunas terapéuticas puede incrementar la eficacia terapéutica de estas. En un estudio exploratorio llevado a cabo por Soriano et al (38), donde se evaluaba la seguridad y eficacia terapéutica de la combinación de QTM con ciclofosfamida y metotrexate junto con racotumomab, se incluyeron 21 pacientes las cuales habían sido tratadas previamente con al menos una línea de quimioterapia para su enfermedad metastásica. En este estudio se obtuvo una RO en el $23,8 \%$ de las pacientes y la mediana de duración de la respuesta terapéutica fue de 18,4 meses. La mediana de SG fue de 12,9 meses (IC 95\%: 7,8-19,9). Se mostró una adecuada tolerancia a la terapia, la mayoría de las reacciones fueron grado 1 .

El vandetanib, un inhibidor múltiple de la tirosin-kinasa que inhibe a VEGFR-2 y EGFR, fue evaluado en 20 pacientes en un ensayo fase I junto con la ciclofosfamida y metotrexate. Los pacientes recibieron vandetanib de forma diaria con tres aumentos de la dosis en grupos cohortes, la ciclofosfamida fue administrada a $50 \mathrm{mg} /$ día y el metotrexate a 2,5 mg dos veces por semana. En este estudio se obtuvo un $\mathrm{BC}$ de $25 \%{ }^{(39)}$.

Selección de los candidatos ideales a recibir quimioterapia metronómica

Según las últimas actualizaciones de las guías ESMO para el manejo del CMM ${ }^{(40,41)}$, se recomienda la QTM como opción terapéutica para pacientes que no requieren una respuesta rápida, aunque es necesario la realización de ensayos clínicos que comparen esquemas metronómicos con esquemas estándares.

En un documento recientemente publicado ${ }^{(13)}$, un grupo de expertos recomen- 
dó su uso en pacientes con enfermedad metastásica con $\mathrm{RH}$ positivos, luego de progresión a una primera línea de tratamiento hormonal. No obstante, otro grupo está en desacuerdo con el mismo por la poca disponibilidad de estudios clínicos que comparen pacientes con $\mathrm{RH}$ negativos versus positivos. En el grupo de pacientes con cáncer de mama triple receptor negativo (CMTN), este mismo grupo de expertos plantean que no existen datos suficientes para avalar su uso en este subgrupo.

Con respecto al uso de la QTM en pacientes con CMTN, Montagna et al ${ }^{(42)}$, en un estudio fase II con 22 pacientes evaluó la eficacia de la vinorelbina junto a la capecitabina y ciclofosfamida como primera línea de tratamiento para enfermedad metastásica. Los resultados de eficacia mostraron una $\mathrm{RO}$ en un $27 \%$ de los pacientes y una SLP a 4 meses de $73 \%$. Se mostró una adecuada tolerancia, registrándose solamente 2 eventos adversos de gravedad.

\section{CONCLUSIONES}

Dada la escasa toxicidad de la QTM y la posibilidad de administración a largo plazo, su espectro de acción es muy amplio y nuevas investigaciones deberán realizarse para esclarecer su verdadero papel ya sea en monoterapia o combinado a otras terapias incluyendo las más novedosas inmunoterapias. No obstante, a pesar de la limitada evidencia científica que soporta el uso de la misma y los controversiales resultados, donde la mayoría de los estudios pertenecen a series de casos, el mayor beneficio clínico pudiera estar dado en el subgrupo de pacientes con baja carga tumoral y estado general aceptable, independientemente del estado de los $\mathrm{RH}$. En este tipo de pacientes debiera definirse el tipo de fármaco y/o esquema de combinación más efectivo, tiempo óptimo para el inicio de la terapia, así como la duración de la misma y los factores a tener en cuenta para la suspensión de la terapia o cambio de agente de combinación.

\section{REFERENCIAS BIBLIOGRÁFICAS}

1. Munzone E, Colleoni M. Metronomic in the neoadjuvant and adjuvant treatment of breast cancer. Cancer Lett. 2017;400:259-266. DOI: 10.1016/j.canlet.2016.12.041
2. Kerbel RS, Shaked Y. The potencial promise of multimodality metronomic chemotherapy revealed by preclinical studies of metastatic disease. Cancer Lett. 2017;400:293-304. DOI: 10.1016/j. canlet.2017.02.005

3. Bocci G, Kerbel RS. Pharmacokinetics of metronomic chemotherapy: a neglected but crucial aspect. Nat Rev Clin Oncol. 2016;13(11):659-673. DOI:10.1038/nrclinonc.2016.64

4. Cazzaniga ME, Biganzoli L, Cortesi L, De Placido $S$, Donadio M, Fabi A, et al. Treating advanced breast cancer with metronomic chemotherapy: what is known, what is new and what is the future? Oncotarget and Therapy. 2019;12:2989-2997. DOI: 10.2147/OTT.S189163.

5. Liu Y, Gu F, Liang J, Dai X, Wan C, Hong X, et al. The efficacy and toxicity profile of metronomic chemotherapy for metastatic breast cancer: a meta-analysis. PLoS One. 2017;12(3):e0173693. DOI:10.1371/journal.pone.0173693.

6. Kontani K, Hashimoto SI, Murazawa C, Norimura S, Tanaka H, Ohtani M, et al. Indication of metronomic chemotherapy for metastatic breast cancer: clinical outcome and responsive subtypes. Mol Clin Oncol. 2016;4(6):947-953. DOI: 10.3892/mco.2016.841

7. Colleoni M, Rocca A, Sandri MT, Zorzino L, Masci $G$, Nolé F, et al. Low-dose oral methotrexate and cyclophosphamide in metastatic breast cancer: antitumor activity and correlation with vascular endothelial growth factor levels. Ann Oncol. 2002;13(1):73-80. DOI: 10.1093/annonc/mdf013

8. Ghiringhelli F, Menard C, Puig PE, Ladoire S, Roux $\mathrm{S}$, Martin F, et al. Metronomic cyclophosphamide regimen selectively depletes $\mathrm{CD} 4+\mathrm{CD} 25+$ regulatory $T$ cells and restores $T$ and NK effector functions in end stage cancer patients. Cancer Immunol Immunother. 2007;56(5):641-648. DOI: 10.1007/ s00262-006-0225-8

9. Gebbia V, Boussen H, Valerio MR. Oral metronomic cyclophosphamide with and without methotrexate as palliative treatment for patients with metastatic breast carcinoma. Anticancer Research. 2012;32(2): 529-536.

10. Soriano JL, Lima M, González J, Batista N, López MV, Rodríguez M, et al. Quimioterapia metronómica con ciclofosfamida y metotrexato en pacientes con cáncer de mama metastásico en progresión. Rev Cub Med. 2009;49(2).

11. Mahmound M, Mohamed R, Mostafa A, Abdallah W, Mohamed N, Eid S, et al. Efficacy and toxicity of metronomic chemotherapy in metastatic breast cancer: egyptian experience. Clin Breast Cancer. 2017;17(8): 618-628. DOI:10.1016/j. clbc.2017.05.001

12. Krajnak S, Battista M, Brenner W, Almstedt K, Elger T, Heimes AS, et al. Explorative analysis of low dose metronomic chemotherapy with cyclophosphamide and methrotrexate in a cohort of metastatic breast cancer patients. Breast Care. 2018;13(2): 272-276. DOI: 10.1159/000487629

13. Cazzaniga ME, Munzone E, Bocci G, Afonso N, Gomez P, Langkjer S, et al. Pan-european expert meeting on the use of metronomic chemotherapy in advanced breast cancer patients: the PENELOPE Project. Adv Ther. 2019;36(2):381-406. DOI: 10.1007/s12325-018-0844-4

14. Addeo R, Sgambato A, Cennamo G, Montella L, Faiola V, Abbruzzese A, et al. Low-dose metronomic oral administration of vinorelbine in first-line treatment of elderly patients with metastatis breast cancer. Clin Breast Cancer. 2010;10(4): 301-306. DOI: 10.3816/CBC.2010.n.039
15. Fedele $P$, Marino A, Orlando L, Achiavone $P$, Nacci A, Sponziello F, et al. Efficacy and safety of lowdose metronomic chemotherapy with capecitabine in heavily pretreated patients with metastatic breast cancer. Eur J Cancer. 2012;48(1):24-29. DOI: 10.1016/j.ejca.2011.06.040

16. Stockler MR, Harvey VJ, Francis PA, Byrne MJ, Ackland SP, Fitzharris B, et al. Capecitabine versus classical cyclophosphamide, methotrexate, and fluorouracil as first-line chemotherapy for advanced breast cancer. J Clin Oncol. 2011; 29(34):44984504. DOI: 10.1200/JCO.2010.33.9101

17. Taguchi T, Nakayama T, Masuda N, Yoshidome K, Akagi K, Nishida Y, et al. Study of low-dose capecitabine monotherapy for metastatic breast cancer. Chemotherapy. 2010;56(2):166-170. DOI:10.1159/000313531

18. Saridaki Z, Malamos N, Kourakos P, Polyzos A, Ardavanis A, Androulakis N, et al. A phase I trial of oral metronomic vinorelbine plus capecitabine in patients with metastatic breast cancer. Cancer Chemother Pharnacol. 2012;69(1): 35-42. DOI: 10.1007/s00280-011-1663-3

19. Cazzaniga ME, Torri V, Villa F, Giuntini N, Riva F, Zappellini A, et al. Vinorelbine and capecitabine in locally advanced or metastatic breast cancer patients: the phase I-II VICTOR-1 study. Inter J Breast Cancer. 2014;2014:769790 DOI:10.1155/2014/769790

20. Cazzaniga ME, Cortesi L, Ferzi A, Scaltriti L, Cicchiello F, Ciccarese M, et al. Metronomic chemotherapy with oral vinorelbine (mVNR) and capecitabine (mCAPE) in advanced HER-2 negative breast cancer: is it a way to optimize disease control? Final results of the VICTOR-2 study. Breast Cancer Res Treat. 2016;160(3):501-509. DOI: 10.1007/s10549-016-4009-3

21. Montagna E, Palazzo A, Maisonneuve P, Cancello G, Iorfida M, Sciandivasci A, et al. Safety and efficacy study of metronomic vinorelbina, cyclophosphamide plus capecitabina in metastatic breast cancer: a phase II trial. Cancer Letter. 2017;400: 276-281. DOI: 10.1016/j.canlet.2017.01.027

22. Montagna E, Vingiani A, Maisonneuve $P$, Cancello G, Contaldo F, Pruneri, et al. Unfavorable prognostic role of tumor-infiltrating lymphocytes in hormone-receptor positive, HER2 negative metastatic breast cancer treated with metronomic chemotherapy. Breast. 2017;34: 83-88. DOI:10.1016/j. breast.2017.05.009

23. Yoshimoto M, Takao S, Hirata M, Okamoto $Y$, Yamashita S, Kawaguchi Y, et al. Metronomic oral combination chemotherapy with capecitabine and cyclophosphamide: a phase II study in patients with HER2-negative metastatic breast cancer. Cancer Chempther Pharmacol. 2012;70(2):331338. DOI:10.1007/s00280-012-1826-X

24. Addeo R, Sperlongano P, Montella L, Vicenzi B, Carraturo M, Lodice $P$, et al. Protracted low dose of oral vinerelbine and temozolomide with whole-brain radiotherapy in the treatment for breast cancer patients with brain metastases. Cancer Chemother Pharmacol. 2012;70(4):603-609. DOI:10.1007/ s00280-012-1945-4

25. Bottini A, Generali D, Brizzi MP, Fox SB, Bersiga A, Bonardi S, et al. Randomized phase II trial of letrozole and letrozole plus low-dose metronomic oral cyclophosphamide as primary systemic treatment in elderly breast cancer patients, J Clin Oncol. 2006;24(22):3623-3628. DOI: 10.1200/ JCO.2005.04.5773. 
26. Licchetta A, Correale P, Migali C, Remondo C, Francini E, Pascucci A, et al. Oral metronomic chemo-hormonal-therapy of metastatic breast cancer with cyclophosphamide and megestrol acetate, J Chemother. 2010;22(3):201-204. DOI:10.1179/ joc.2010.22.3.201

27. Schwartzberg LS, Wang G, Somer BG, Blakely LJ, Wheeler BJ, Walker MS, et al. Phase II trial of fulvestrant with metronomic capecitabina for postmenopausal women with hormone receptor-positive, HER2-negative metastatic breast cancer. Clin Breast Cancer.2014;14(1):13-19. DOI: 10.1016/j. clbc.2013.09.003

28. Aurilio G, Munzone E, Botteri E, Sciandivasci A, Adamoli L, Minchella I, et al. Oral metronomic cyclophosphamide and methotrexate plus fulvestrant in advanced breast cancer patients: a monoinstitutional case-cohort report. Breast J. 2012;18(5):470-474. DOI: 10.1111/j.15244741.2012.01278.x

29. Cazzaniga ME, Dionisio MR, Riva F. Metronomic chemotherapy for advanced breast cancer patients. Cancer Letter. 2017; 400: 252-258. DOI:10.1016/j.canlet.2016.12.019

30. Kareva I, Waxman DJ, Klement GL. Metronomic chemotherapy: an attractive alternative to maximum tolerated dose therapy that can activate anti-tumor immunity and minimize therapeutic resistance. Cancer Lett. 2015;358(2):100-106. DOI: 10.1016/j.canlet.2014.12.039

31. Chen YL, Chang MC, Cheng WF. Metronomic chemotherapy and immunotherapy in cancer treatment. Cancer Letters. 2017;400:282-292. DOI: 10.1016/j.canlet.2017.01.040
32. Garcia-Sáenz JA, Martín M, Calles A, Bueno C, Rodríguez L, Bobokova J, et al. Bevacizumab in combination with metronomic chemotherapy in patients with anthracycline- and taxane-refractory breast cancer. J Chemother. 2008;20(5): 632-639. DOI: 10.1179/joc.2008.20.5.632

33. Dellapasqua S, Bertolini F, Bagnardi V, Campagnoli E, Scarano E, Torrisi R, et al. Metronomic cyclophosphamide and capecitabine combined with bevacizumab in advanced breast cancer. J Clin Oncol 2008;26(30):4899-4905. DOI:10.1200/ JCO.2008.17.4789.

34. Montagna E, Cancello G, Bagnardi V, Pastrello D, Dellapasqua S, Perri G, et al. Metronomic chemotherapy with bevacizumab and erlotinib in patients with metastatic HER-negative breast cancer: clinical and biological activity. Clin Breast Cancer. 2012;12(3):207-214. DOI: 10.1016/j. clbc.2012.03.008

35. Calleri A, Bono A, Bagnardi V, Quarna J, Mancuso $\mathrm{P}$, Rabascio $\mathrm{C}$, et al. Predictive potential of angiogenic growth factors and circulating endothelial cells in breast cancer patients receiving metronomic chemotherapy plus bevacizumab. Clin Cancer Res. 2009;15(24):7652-7657. DOI: 10.1158/10780432.CCR-09-1493

36. Orlando L, Cardillo A, Ghisini R, Rocca A, Balduzzi $A$, Torrisi $R$, et al. Trastuzumab in combination with metronomic cyclophosphamide and methotrexate in patients with HER-2 positive metastatic breast cancer. BMC Cancer. 2006;6:225. DOI:10.1186/1471-2407-6-225

37. Wildiers H, Tryfonidis K, Dal Lago L, Vuylsteke P, Curigliano G, Waters $S$, et al. Pertuzumab and trastu- zumab with or without metronomic chemotherapy for older patients with HER2-positive metastatic breast cancer (EORTC 75111-10114): an open-label, randomized, phase 2 trial from Elderly Task Force/Breast Cancer Group. Lancet Oncol. 2018;19(3):323-336. DOI: 10.1016/S1470-2045(18)30083-4

38. Soriano JL, Batista N, Santiesteban E, Lima M, González J, Garcia R et al. Metronomic cyclophosphamide and methotrexate chemotherapy combined with $1 \mathrm{E} 10$ anti-idiotype vaccine in metastatic breast cancer. Int J Breast Cancer. 2011;2011: 710292. DOI: 10.4061/2011/710292

39. Mayer EL, Isakoff SJ, Klement S, Downing SR, Chen WY, Hannagan K, et al. Combination antiangiogenic therapy in advanced breast cancer: a phase 1 trial of vandetanib, a VEGFR inhibitor, and metronomic chemotherapy, with correlative platelet proteomics. Breast Cancer Res Treat. 2012; 136(1):169-178. DOI: 10.1007/s10549-012-2256-5

40. Cardoso F, Senkus E, Costa A, Papadopoulos E, Aapro M, André F, et al. $4^{\text {th }}$ ESO-ESMO international consensus guidelines for advanced breast cancer (ABC4). Ann Oncol. 2018,29(8):1634-1657. DOI: 10.1093/annonc/mdy192

41. Cardoso F, Costa A, Senkus E, Aapro M, Andre $\mathrm{F}$, Barrios $\mathrm{CH}$, et al. $3^{\text {rd }}$ ESO-ESMO international consensus guidelines for advanced breast cancer (ABC3). Ann Oncol. 2017;28(12):3111. doi 10.1093/annonc/mdx036.

42. Montagna E, Bagnardi V, Cancello G, Sangalli C, Pagan E, Iorfida M, et al. Metronomic chemotherapy for first-line treatment of metastatic triple negative breast cancer: a phase II trial. Breast Care. 2018:13(2):177-181 DOI: 10.1159/000487630 